\title{
Low-energy excitations and the fast process of polystyrene thin supported films studied by inelastic and quasielastic neutron scattering
}

\section{$\operatorname{AUTHOR}(\mathrm{S})$ :}

Inoue, R; Kanaya, T; Nishida, K; Tsukushi, I; Shibata, $\mathrm{K}$

\section{CITATION:}

Inoue, R ... [et al]. Low-energy excitations and the fast process of polystyrene thin supported films studied by inelastic and quasielastic neutron scattering. PHYSICAL REVIEW E 2006, 74(2): 021801.

\section{ISSUE DATE:}

2006-08

URL:

http://hdl.handle.net/2433/39916

RIGHT:

Copyright 2006 American Physical Society 
PHYSICAL REVIEW E 74, 021801 (2006)

\title{
Low-energy excitations and the fast process of polystyrene thin supported films studied by inelastic and quasielastic neutron scattering
}

\author{
Rintaro Inoue, ${ }^{1}$ Toshiji Kanaya, ${ }^{1, *}$ Koji Nishida, ${ }^{1}$ Itaru Tsukushi, ${ }^{2}$ and Kaoru Shibata ${ }^{3}$ \\ ${ }^{1}$ Institute for Chemical Research, Kyoto University, Uji, Kyoto-fu 611-0011, Japan \\ ${ }^{2}$ Chiba Institute of Technology, Narashino, Chiba-ken 275-0023, Japan \\ ${ }^{3}$ Japan Atomic Energy Research Institute, Tokai, Ibaraki-ken 319-1195, Japan
}

(Received 1 March 2006; published 3 August 2006)

\begin{abstract}
We studied the low-energy excitations in the meV region as well as the picosecond fast process in polystyrene thin supported films using inelastic and quasielastic neutron scattering in a temperature range from 11 to $430 \mathrm{~K}$, covering the bulk glass transition temperature $T_{\mathrm{g}}$. It was found that the mean square displacement $\left\langle u^{2}\right\rangle$ decreased with the film thickness below and above the glass transition temperature $T_{\mathrm{g}}$, suggesting that the hardening occurs with decreasing the film thickness. Corresponding to the decrease in $\left\langle u^{2}\right\rangle$, it was also found that the density of phonon states $G(\omega)$ decreased with film thickness. This decrease occurs mainly in the Debye mode while the contribution of the boson peak mode also decreases with the film thickness without changing the boson peak energy. As for the fast process, which appeared above at about $150 \mathrm{~K}$ as a quasielastic scattering, the fraction $A_{\text {fast }}(Q)$ decreased with the film thickness in a similar manner with the boson peak without changing the relaxation rate, suggesting a common origin for the boson peak and the fast process. These observations showing the hardening were well explained by assuming a hard dense layer at the interface, where the numbers of the boson peak mode as well as the fast process are less than the bulk state.
\end{abstract}

DOI: 10.1103/PhysRevE.74.021801

PACS number(s): 82.35. $-\mathrm{x}, 68.60 .-\mathrm{p}, 61.12 . \mathrm{Ex}, 63.50 .+\mathrm{x}$

\section{INTRODUCTION}

Polymer thin films are utilized in various industrial fields because their properties are related to important phenomena such as dewetting, a lubricant, photolithography, and so on. Therefore, polymer thin films have been extensively studied by many researchers not only from an industrial point of view but also from a science point of view $[1,2]$. One of the most interesting topics in polymer thin film studies is the thickness dependence of the glass transition temperature $T_{\mathrm{g}}$ $[3,4]$. In the case of polystyrene (PS), $T_{\mathrm{g}}$ decreases with film thickness for both thin films supported on a Si substrate [5] and free standing thin films [6-8] as revealed by various methods such as ellipsometry [9], x-ray and neutron reflectivity [10-12], positron lifetime spectroscopy (PALS) [13], dielectric relaxation $[14,15]$, Brillouin light scattering $[6,8]$, atomic force microscopy [16-18], calorimetry $[19,20]$, and low-energy muons [21]. Another interesting finding is the thickness dependence of thermal expansivity. Orts et al. [22] first reported that the film thickness decreases in the glassy state with increasing temperature in a thickness range below about $25 \mathrm{~nm}$. Recently, Miyazaki et al. [10,11] have revealed that this apparent negative expansivity in the glassy state was caused by a lack of annealing. They also showed that the expansivity decreases with film thickness even after a long annealing above the glass transition temperature, suggesting that the smaller expansivity in thin films than in bulk is inherent. The origin of the small thermal expansivity in the glassy state in thin films has been discussed on the basis of inelastic neutron scattering data [23]. This study as well as other dynamical studies [14-18,24-29] demonstrate that dy-

\footnotetext{
*Corresponding author. FAX: +81-774-38-3146. Email address: kanaya@scl.kyoto-u.ac.jp
}

namical researches are essential to understand the glass transition and the related phenomena. Inelastic and quasielatic neutron scattering provides unique information on fast and local dynamics of polymer thin films although measurements on polymer thin films are very hard due to the very weak scattering intensity [26-29].

In the previous work [29] we have studied dynamics of polystyrene thin supported films in glassy states in the meV region and found that the mean square displacement $\left\langle u^{2}\right\rangle$ decreased with the film thickness, suggesting the hardening of polystyrene films with decreasing the film thickness. In this work we have extended inelastic and quasielastic neutron scattering measurements on polystyrene thin supported films to a higher temperature region above the bulk glass transition temperature, and discuss the mean square displacement $\left\langle u^{2}\right\rangle$, the low-energy excitations including the boson peak in the meV region, and the so-called picosecond fast process in order to understand the origin of the hardening of PS thin films with decreasing the film thickness.

\section{EXPERIMENT}

We used PS with molecular weight $M_{w}=2.9 \times 10^{5}$ and molecular weight distribution $M_{w} / M_{n}=1.06$, where $M_{w}$ and $M_{n}$ are the weight average and the number average of the molecular weight, respectively. The bulk glass transition temperature $T_{\mathrm{g}}$ determined by a differential scanning calorimetry (DSC) measurements was $373 \mathrm{~K}$. PS thin films were prepared by spin-coating toluene solutions at $2000 \mathrm{rpm}$ on flat glass plates rinsed in toluene prior to the spin coating. The film thickness was controlled by varying the concentration of PS in solution and we prepared films 1000 and $400 \AA$ thick. The film was removed from the glass plate onto the water surface and then collected on $\mathrm{Al}$ foil $15 \mu \mathrm{m}$ thick, and 
then annealed at $413 \mathrm{~K}$ for $12 \mathrm{~h}$ after drying in vacuum at room temperature for $2 \mathrm{~d}$. As shown in the previous papers $[10,11]$, this annealing condition is enough to study the glass transition and thermal expansivity of PS thin supoorted films. 299 sheets of the films on $\mathrm{Al}$ foils were rolled up and placed into a hollow cylindrical $\mathrm{Al}$ cell $14 \mathrm{~mm}$ in diameter and $45 \mathrm{~mm}$ high in order to get enough inelastic scattering intensity. A bulk PS sample with a thickness of $0.1 \mathrm{~mm}$ was prepared as a control sample.

The inelastic neutron scattering measurements were performed with an inverted geometry time of flight (TOF) spectrometer LAM-40 [30] installed at the cold spallation neutron source in High Energy Accelerator Research Organization (KEK), Tsukuba, Japan. In the spectrometer, the final energy of neutron and the energy resolution was 4.59 and $\sim 0.2 \mathrm{meV}$ at the elastic position, respectively. The measurements were carried out at temperatures from 11 to $430 \mathrm{~K}$, covering the bulk glass transition temperature $T_{\mathrm{g}}$ of $373 \mathrm{~K}$. The observed TOF spectra of the 299 thin films on Al foilds and the empty can including $299 \mathrm{Al}$ foils were corrected for the self-shielding, the counter efficiency and the incident neutron spectrum at each temperature and the empty can scattering was carefully subtracted from the sample scattering. The PS used in this experiment is a fully protonated one, and the scattering intensity obtained here is mainly dominated by the incoherent scattering because the incoherent atomic scattering cross section of a hydrogen atom is much larger than coherent cross sections of hydrogen and incoherent and/or cohernet cross sections of carbon atom.

\section{RESULTS AND DISCUSSION}

Figures 1(a) and 1(b) indicate the dynamic scattering laws $S(Q, \omega)$ of the films 400 and $1000 \AA$ thick at temperatures from 11 to $408 \mathrm{~K}$, respectively, which were obtained by summing up 6 spectra from $Q=0.87$ to $2.47 \AA^{-1}$. The intensities were normalized to the total scattering intensity at the lowest temperature $11 \mathrm{~K}$. A broad inelastic peak, which is the so-called boson peak [31,32] characteristic to amorphous materials, is observed at around $1.5 \mathrm{meV}$ at low temperatures below about $150 \mathrm{~K}$. With increasing temperature, the shape of the spectrum changes from inelasticlike to quasielasticlike, implying the onset of a relaxation process, which is the so-called picosecond fast process [33-37].

First we analyzed the elastic scattering intensity $I_{\mathrm{el}}(Q)$ to evaluate the mean square displacement $\left\langle u^{2}\right\rangle$. It is known that the $Q$ dependence of incoherent elastic intensity $I_{\mathrm{el}}(Q)$ is related to $\left\langle u^{2}\right\rangle$ through $I_{\mathrm{el}}(Q) \sim \exp \left[-\left\langle u^{2}\right\rangle Q^{2}\right]$. According to this relation we plotted the elastic scattering intensity $I_{\mathrm{el}}(Q)$ against $Q^{2}$. In Fig. 2, an example of such a plot is shown for the bulk and the 1000 and $400 \AA$ films at $230 \mathrm{~K}$, where the elastic scattering intensity $I_{\mathrm{el}}(Q)_{230 \mathrm{~K}}$ is divided by $I_{\mathrm{el}}(Q)_{11 \mathrm{~K}}$ at the lowest temperature $11 \mathrm{~K}$ in order to reduce the effect of coherent scattering under an assumption that the structure factor does not change with temperature. Therefore, the observed $\left\langle u^{2}\right\rangle$ corresponds to $\left\langle u^{2}\right\rangle_{230 \mathrm{~K}}-\left\langle u^{2}\right\rangle_{11 \mathrm{~K}}$, meaning that the $\left\langle u^{2}\right\rangle$ is zero at $11 \mathrm{~K}$. A similar evaluation was done at

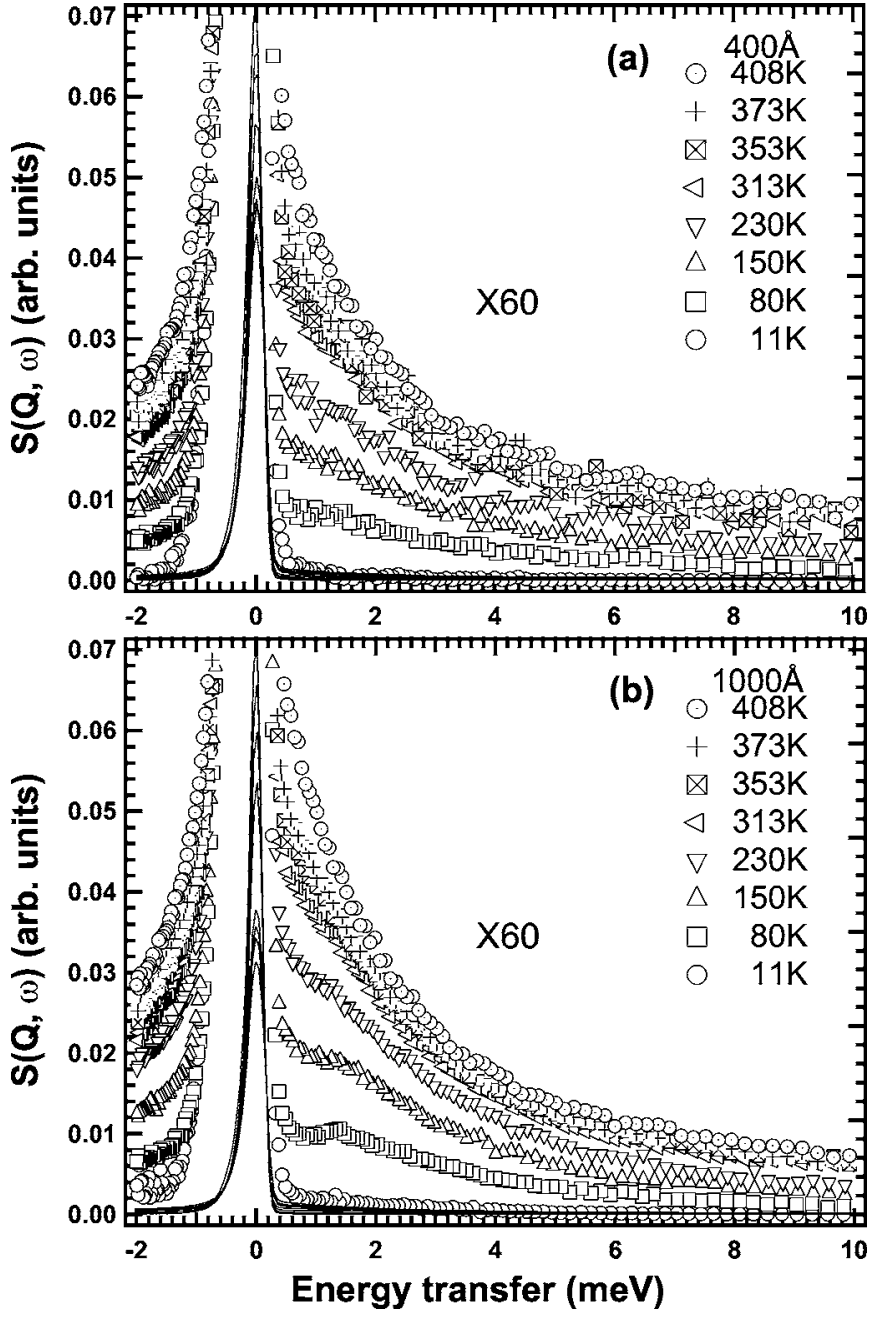

FIG. 1. Dynamic scattering law $S(Q, \omega)$ of PS thin films measured with LAM-40 at various temperatures. (a) $400 \AA$ and (b) $1000 \AA$. Solid curves show whole shapes of the spectra at each temperature and symbols are the expanded ones by a factor of 60 .

each temperature and the temperature dependence of $\left\langle u^{2}\right\rangle$ is shown in Fig. 3 for the bulk and the 1000 and $400 \AA$ thin films. The mean square displacement $\left\langle u^{2}\right\rangle$ is almost proportional to $T$ in the low temperature range below about $150 \mathrm{~K}$

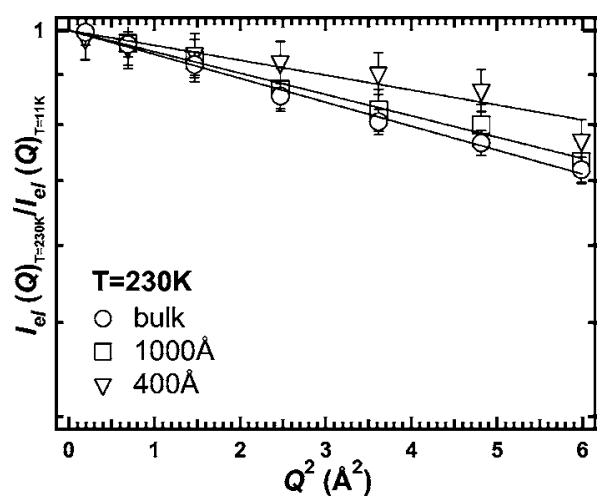

FIG. 2. $Q^{2}$ dependence of elastic intensity $I_{\mathrm{el}}(Q)$ at $230 \mathrm{~K}$ divided by that at $11 \mathrm{~K}$ : bulk $(\bigcirc), 1000 \AA(\square)$, and $400 \AA(\nabla)$. 


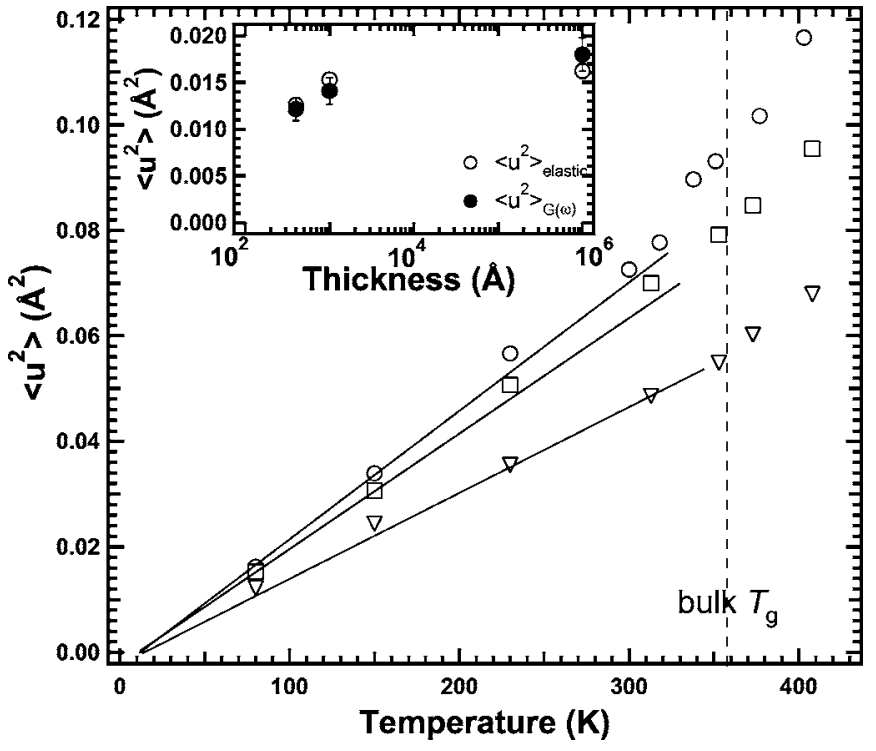

FIG. 3. Temperature dependence of a mean square displacemment $\left\langle u^{2}\right\rangle$ : bulk $(\bigcirc), 1000 \AA(\square)$, and $400 \AA(\nabla)$. Dashed line shows the bulk glass transition temperature $T_{\mathrm{g}}$. Inset shows the film thickness dependendence of $\left\langle u^{2}\right\rangle_{\text {elastic }}$ evaluated from the elastic scattering intensity $(O)$ and $\left\langle u^{2}\right\rangle_{\mathrm{G}(\omega)}$ calculated from the density of phonon states at $80 \mathrm{~K}$.

as indicated by solid lines in the figure, showing harmonic nature of the vibration. The solid lines were drawn so as to go through $\left\langle u^{2}\right\rangle=0$ at $T=11 \mathrm{~K}$. As the temperature further increases, the mean square displacement begins to deviate from the linear relationship and shows excess value over the harmonic contribution. This anharmonic contribution is caused by the onset of the picosecond fast process. The deviation temperature lies at around $150 \mathrm{~K}$ for bulk [34,35] and the $1000 \AA$ film, but seems to shift to higher temperatures for the $400 \AA$ film, although the exact evaluation is not easy. It should be noted that the glass transition temperature $T_{\mathrm{g}}$ is not detected in the measurements because the so-called $\alpha$ process is not observed with the present energy resolution $(\delta E$ $\sim 0.2 \mathrm{meV}$ ). In the whole temperature range examined the mean square displacement $\left\langle u^{2}\right\rangle$ decreases with the film thickness. As an example, the mean square displacement $\left\langle u^{2}\right\rangle$ at $80 \mathrm{~K}$, which is in the harmonic temperature range, is plotted against the film thickness in the inset of Fig. 3. The mean square displacement $\left\langle u^{2}\right\rangle$ of harmonic vibration is related to the density of phonon states $G(\omega)$ through the following relation [38]:

$$
\left\langle u^{2}\right\rangle=\frac{3 \hbar}{2 M} \int_{0}^{\infty} \frac{1}{\omega} \operatorname{coth}\left(\frac{\hbar \omega}{2 k_{B} T}\right) G(\omega) d \omega .
$$

The decrease in $\left\langle u^{2}\right\rangle$ with the film thickness below about $150 \mathrm{~K}$ suggests that the density of phonon states $G(\omega)$ is shifted to a high-energy side or reduced in a low-energy side. In other words, the hardening of a vibration mode occurs with decreasing the PS film thickness.

In order to see the origin of the hardening we analyze the inelastic scattering part of the thin films. The observed

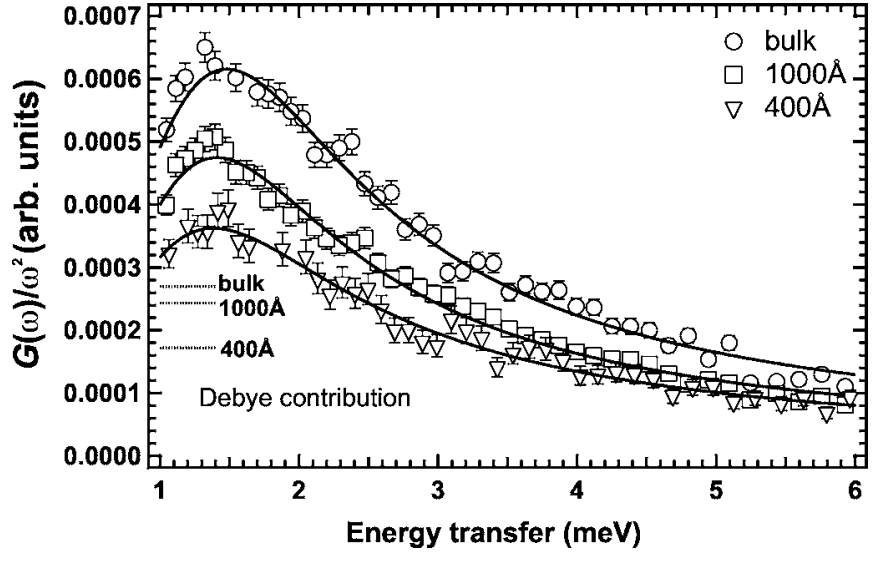

FIG. 4. Density of phonon states $G(\omega)$ divided by $\omega^{2}$ : bulk $(\bigcirc)$, $1000 \AA(\square)$, and $400 \AA(\nabla)$. Dotted lines show the Debye contributions $G_{\mathrm{D}}(\omega) / \omega^{2}$.

$S(Q, \omega)$ at $80 \mathrm{~K}$ was converted to the density of phonon states $G(\omega)$ after subtracting the elastic contribution and correcting for the Debye-Waller factor. For the subtraction, the elastic line was reconstructed from the sample scattering at $11 \mathrm{~K}$ because the shape of the elastic line is affected by the sample shape, and in addition the inelastic scattering at $11 \mathrm{~K}$ was evaluated from the spectrum at $80 \mathrm{~K}$. The Debye-Waller factor used in the calculation of $G(\omega)$ was evaluated from the elastic intensity. The evaluated $G(\omega) / \omega^{2}$ are shown in Fig. 4 for the bulk and the 1000 and $400 \AA$ films. It is clear that the $G(\omega)$ decreases with the film thickness in the energy region of $1-10 \mathrm{meV}$, suggesting that the decrease in $\left\langle u^{2}\right\rangle$ is caused by the decrease in $G(\omega)$. Using the observed $G(\omega)$ we have calculated $\left\langle u^{2}\right\rangle$ for the bulk and the 1000 and $400 \AA$ films at $80 \mathrm{~K}$ through Eq. (1) with $M=104$ (monomer mass). The absolute value of $G(\omega)$ was evaluated by calculating the heat capacity from $G(\omega)$ and comparing it to the measured one [39], and was smoothly extrapolated to $3 / \omega_{\mathrm{D}}{ }^{3}$ at $0 \mathrm{meV}$, where $\omega_{\mathrm{D}}$ is the Debye frequency calculated from the sound velocity obtained in Brillouin scattering. In the calculation we took into account contributions from the Debye mode and the boson peak mode, and the contribution of the Debye mode in $G(\omega)$ was evaluated from the Debye frequency $\omega_{D}$. The contribution of the boson peak mode was obtained by subtracting the Debye contribution from the total one. For the thin films the Debye contributions were estimated assuming that the amplitudes of the Debye mode and the boson peak mode are independent of film thickness. The calculated $\left\langle u^{2}\right\rangle$ was plotted in the inset of Fig. 3 as a function of film thickness. It is clear that the reduction in $\left\langle u^{2}\right\rangle$ could be attributable to the reduction in $G(\omega)$.

We now consider which mode is hardened or reduced with decreasing the film thickness in the low-energy region below about $10 \mathrm{meV}$. In this energy region there exist at least two modes [31]: the localized mode (boson peak mode) and the extended mode (Debye mode). As seen in Fig. 4, the boson peak energy (or frequency) lies at around $1.5 \mathrm{meV}$ for all the samples and the peak position is independent of film thickness within the experimental error. If we assume the peak energy is given by $\omega=\sqrt{f / m}$, where $f$ and $m$ are the 
harmonic force constant and the effective mass, the present result means that the hardening of the boson peak mode is very small or negligible. Nevertheless, we observed the reduction in $G(\omega)$ with the film thickness. The Debye contribution $G_{\mathrm{D}}(\omega)$ to the density of phonon states was calculated for the bulk and the thin films under an assumption that the amplitude of the two modes are independent of film thickness and indicated in Fig. 4 by dashed lines, showing the clear decrease in $G_{\mathrm{D}}(\omega)$. We also evaluated the contribution of the boson peak mode $G_{\mathrm{B}}(\omega)$ to the density of phonon states by subtracting the Debye contribution $G_{\mathrm{D}}(\omega)$ from the total one, and found that $G_{\mathrm{B}}(\omega)$ also decreased with film thickness although the boson peak energy is unaltered with the film thickness. Therefore, the observed decrease in $\left\langle u^{2}\right\rangle$ could be attributed to the decrease in the density of phonon states in both the Debye mode and the boson peak mode.

In a previous paper [29] we discussed two molecular pictures for the hardening with decreasing the film thickness. One is the hardening due to the polymer chain confinement in a thin film. In a thin film below polymer coil size, which can be represented by twice the radius of gyration $\left(2 R_{\mathrm{g}}\right)$, polymer chains could not sustain the normal coil form and the deformation would occur. Such deformed polymer chains would have a higher restoring force than that of normal coil, resulting in the increase of $f$ with decreasing film thickness. In fact, the $R_{\mathrm{g}}$ of PS used in this experiment is about $150 \AA$ [40] and the twice of $R_{\mathrm{g}}$ is about $300 \AA$. Taking into account the distribution of polymer coil sizes, the confinement effects may be expected for the $400 \AA$ film, but not for the $1000 \AA$ film. Nevertheless the reduction in $\left\langle u^{2}\right\rangle$ was observed even in the $1000 \AA$ film. This implies another possibility for the hardening. A hard layer at the interface, which is often called "dead layer" [13], is also a candidate for the hardening. If we assume such a hard layer with constant thickness independent of total film thickness at the interface, it is expected that the average force constant increases with decreasing the total film thickness because the fraction of the hard layer increases with decreasing the film thickness. Assuming $\left\langle u^{2}\right\rangle=0$ in the hard layer, we estimated that the thickness of the hard layer was $\sim 110 \AA$, which seems rather large but still in a range of reported values for the hard layer [13,14,27]. We would like to discuss which possibility is more plausible to explain the decrease in the density of phonon states of both the Debye mode $G_{\mathrm{D}}(\omega)$ and the boson peak mode $G_{\mathrm{B}}(\omega)$.

The density of phonon states $G_{\mathrm{D}}(\omega)$ of the Debye mode is related to the average sound velocity $v$ through the relation [31] $G_{\mathrm{D}}(\omega)=4 \pi V \omega^{2} / v^{3}$, where $V$ is the average atomic volume. One possibility for the reduction in the Debye mode is the cutoff of the long wavelength phonon due to the confinement. Zorn and co-workers explained the decrease of the low frequency mode in salol and polydimethylsiloxane (PDMS) in porous glass in terms of the cutoff of the phonon with wavelength longer than the pore size $[41,42]$. We evaluated the sound velocity of PS at $80 \mathrm{~K}$ based on the generalized Rao function presented by Schuyer [43]. The cutoff energy (or frequency) using the procedure by Zorn et al. for the $400 \AA$ PS thin films was calculated to be about $95 \mu \mathrm{eV}$. This cutoff energy is located within the elastic scattering in this experiment $(\delta E \sim 0.2 \mathrm{meV})$, therefore the cutoff of the long wavelength phonon is not the cause for the reduction in the inelastic intensity above $0.2 \mathrm{meV}$ with film thickness. Another possibility for the reduction in $G_{\mathrm{D}}(\omega)$ is the increase of sound velocity $(v)$ due to the densification with decreasing film thickness. In fact, it was reported that polymer chains tend to form ordered structure exhibiting layering [44] near the interface that is related to the radius of the gyration of polymers [45] as pointed out by Mukherjee et al. [46]. The density of layers at the interface must become larger due to such ordered structure, resulting in the increase in sound velocity with decreasing the film thickness. This ordered layer must act as the hard layer (or the dead layer), resulting in the decrease of the Debye contribution $G_{\mathrm{D}}(\omega)$. It is worthnoting that no signs of the free surface were observed in the measurements, to which the reduction in $T_{\mathrm{g}}$ is often assigned. One possibility is that the free surface motion is too slow to be detected with the present energy resolution $\delta E$ $\sim 0.2 \mathrm{meV}$.

The hard layer at the interface could explain the decrease in the boson peak contribution $G_{\mathrm{B}}(\omega)$ as well. The densification near the interface leads to the decrease in the number of defects or voids. If the origin of the boson peak concerns defects or voids [47-50], the decrease of the defects or voids leads to the decrease in the number of the boson peak mode, resulting in thee decreases of $G_{\mathrm{B}}(\omega)$, which is a kind of mode reorganization. The concept of chain confinement can explain the decrease of the Debye mode while it seems hard to explain the decrease in the boson peak mode. Hence, the hard layer or the dead layer seems more plausible to explain the decrease in the density of phonons than the chain confinement in a thin film.

Next, we will discuss the quasielastic scattering due to the picosecond fast process in a temperature range above about $150 \mathrm{~K}$. The observed dynamic scattering law $S(Q, \omega)$ was scaled by the Bose factor after the correction for the DebyeWaller factor using $\left\langle u^{2}\right\rangle$ evaluated from the $Q^{2}$ dependence of the elastic scattering. The scaled spectra are shown in Fig. 5 for the 1000 and $400 \AA$ films, where the reference temperature is $80 \mathrm{~K}$. The spectra were well scaled in the energy range above about $1.5 \mathrm{meV}$ including the boson peak, while below $1.5 \mathrm{meV}$ the excess scattering was pronounced with increasing temperature above about $200 \mathrm{~K}$. This suggests the onset of the so-called fast process [33-37]. In order to see the deviation from the Bose scaling, we integrated the scaled spectrum in the quasielastic region $(0.70$ to $1.5 \mathrm{meV})$ avoiding the effect of elastic scattering and plotted against the temperature in Fig. 6 for the bulk and the $1000 \AA$ and $400 \AA$ films. It is hard to estimate the deviation temperature because the data points are rather little and the experimental error is large. We assumed that the deviation for the bulk started at around $150-200 \mathrm{~K}$ as reported in the previous works [34], and that the excess scattering was not yet observed at $150 \mathrm{~K}$ as indicated by solid lines in Fig. 6. From the figure we can say that the temperature dependence of the integrated intensity in the $400 \AA$ thin film is almost flat compared to the bulk and the $1000 \AA$ thin films in a low temperature region below $\sim 230 \mathrm{~K}$. This qualitatively corresponds to the higher deviation temperature in $\left\langle u^{2}\right\rangle$ for the $400 \AA$ film than the bulk and the $1000 \AA$ film. The results sugget that the quasielastic scat- 


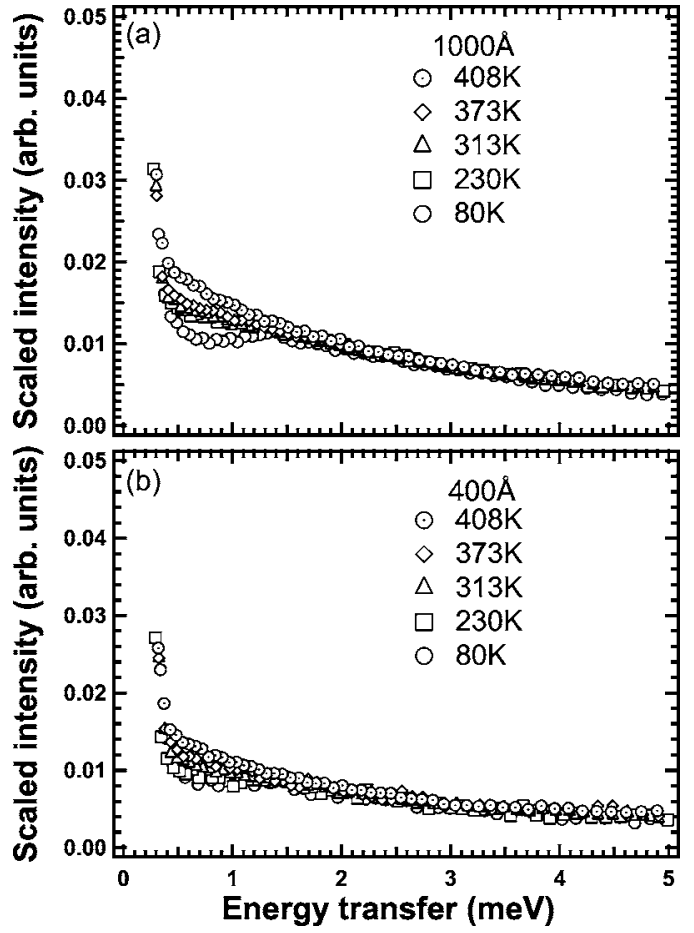

FIG. 5. Bose-scaled inelastic scattering intensity after correcting for the Debye-Waller factor: (a) $1000 \AA$ and (b) $400 \AA$. The reference temperature is $80 \mathrm{~K}$ for both (a) and (b).

tering due to thermal fluctuations becomes harder to occur in the thinner films, agreeing with the picture of hardening.

In order to study the characteristic features of the fast process in more detail, the observed dynamic scattering law $S(Q, \omega)$ was fitted to a model function convoluted with the resolution function of the spectrometer. We employed the following model function which could describe the $S(Q, \omega)$ for the bulk [35]:

$S(Q, \omega)=C(Q)\left[\left\{1-A_{\text {fast }}(Q)\right\} \delta(\omega)+A_{\text {fast }}(Q) L(\Gamma, \omega)\right]+B(Q)$,

where $A_{\text {fast }}(Q)$ is a fraction of the fast process, and the $\delta(\omega)$ and $L(\Gamma, \omega)$ are a $\delta$ function and a Lorentzian with a half-

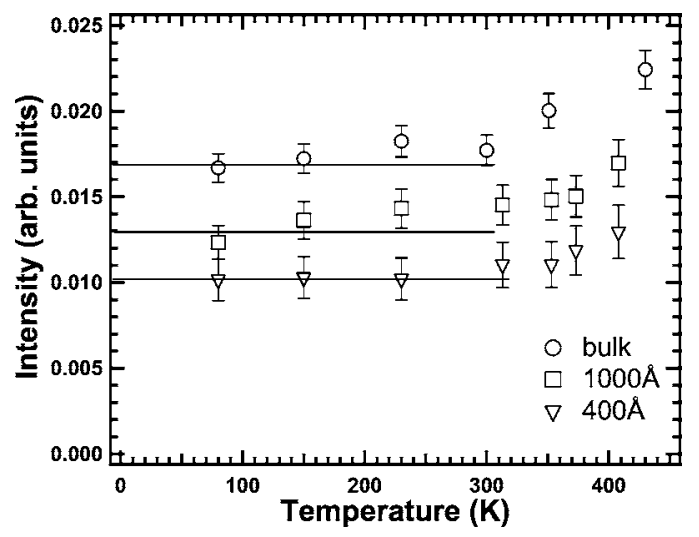

FIG. 6. Temperature dependence of the integrated intensity of the Bose-scaled spectra in Fig. 5 in an energy range from 0.70 to $1.5 \mathrm{meV}$ : bulk $(\bigcirc), 1000 \AA(\square)$, and $400 \AA(\nabla)$.

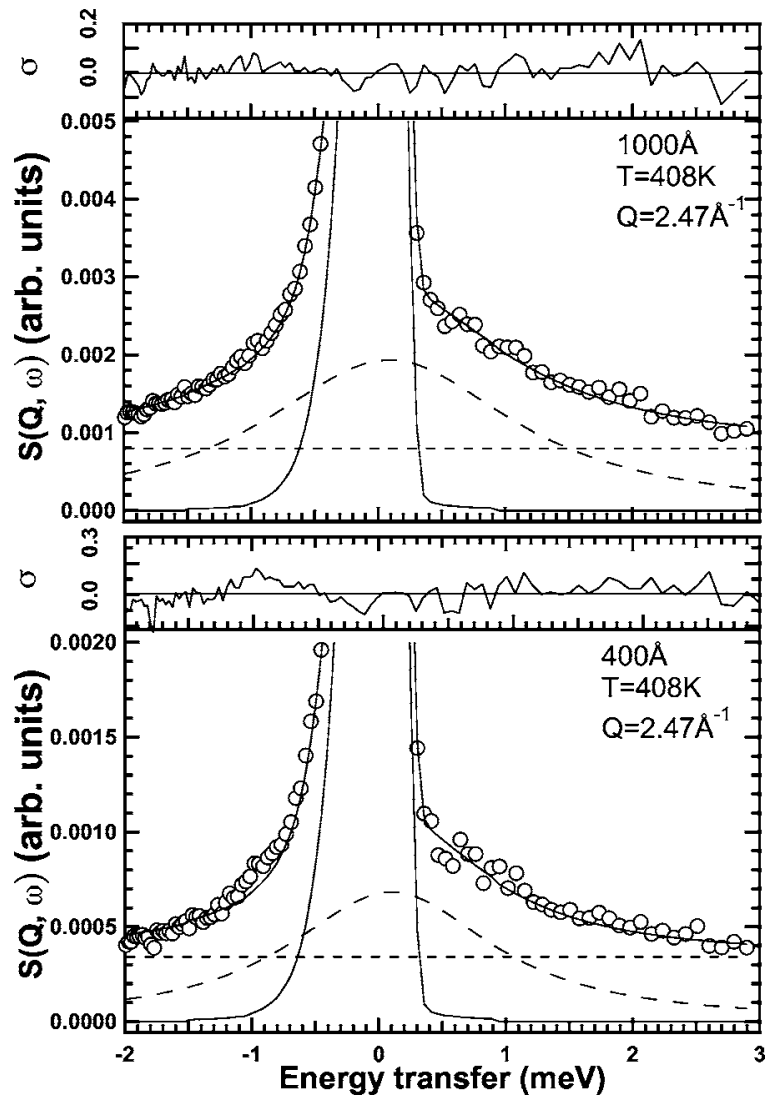

FIG. 7. Results of curve fitting to the LAM-40 spectra of 1000 and $400 \AA$ thin films at $T=408 \mathrm{~K}$ and $Q=2.47 \AA^{-1}$. (一), elastic componetnt; (-), quasielastic Lorentzian; and (---), flat background.

width at half-maximum $\Gamma$, respectively. $C(Q)$ and $B(Q)$ are constants, representing the Debye-Waller factor and inelastic flat background, respectively. It is not clear how to separate the inelastic scattering component from the fast process. In this fit, it was assumed that the boson peak could be included in the inelastic flat component $B(Q)$ at least in the limited energy range below $3 \mathrm{meV}$. A $\delta$ function was introduced to describe immobile parts of polymer chains as well as slow relaxation processes within the energy resolution function such as slow $\beta$ process, $\alpha$ process, and reptation motion [35,51]. The results of the fits for the 1000 and $400 \AA$ films at $408 \mathrm{~K}$ are shown Fig. 7. We found that the fast process of PS thin films were also described by a single Lorentzian as was the case of the bulk [35]. No other relaxation processes were observed even above $T_{\mathrm{g}}$ except for the fast process in the energy region above $\sim 0.2 \mathrm{meV}$, meaning that we could not detect $T_{\mathrm{g}}$ due to the limited energy resolution in this measurement. It is noted that we also performed another analysis, in which we described the Boson peak and the Debye contributions by a spectrum predicted from the $80 \mathrm{~K}$ data by the Bose factor and fitted an additional Lorentzian to the observed $S(Q, \omega)$. The results of the fits were not bad, but we employed the former model including the inelastic flat background in this report because the deviation plot was better than the latter model.

In order to study the nature of the fast process furthermore, logarithms of the relaxation rate $\Gamma$ and the fraction 


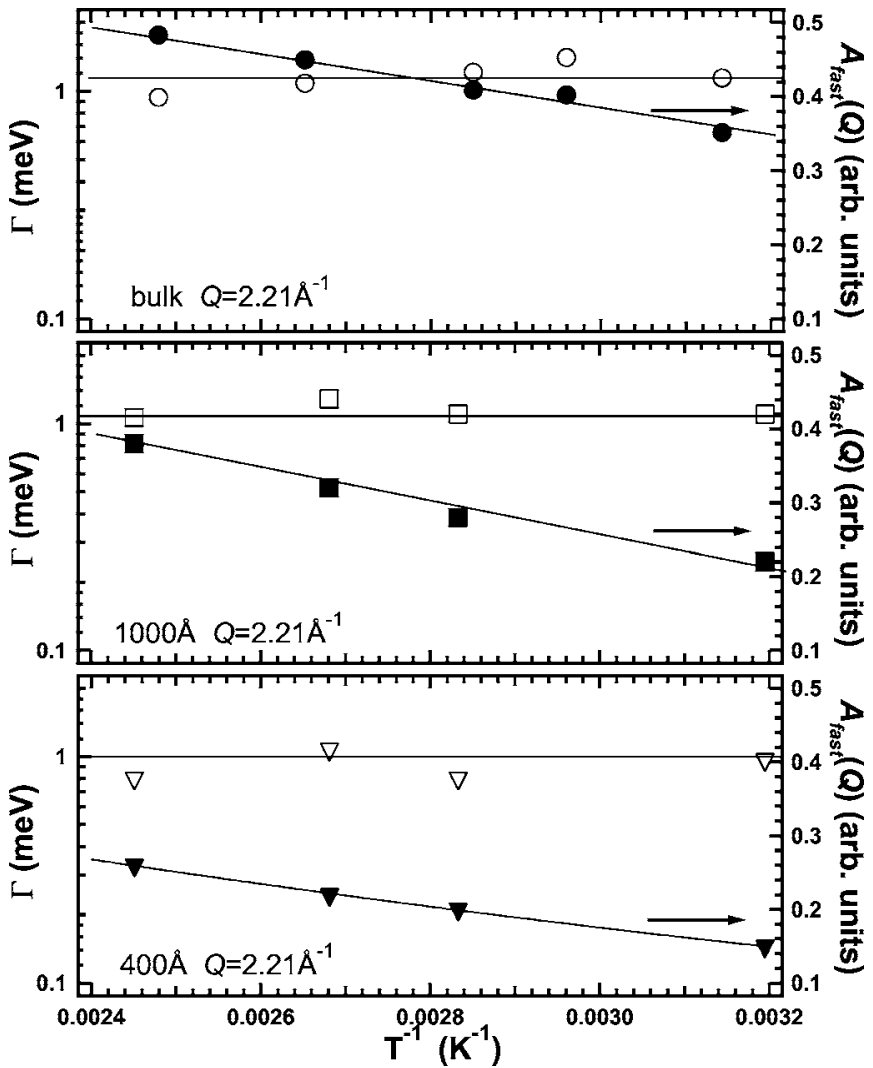

FIG. 8. Temperature dependence of relaxation rate $\Gamma$ and fraction $A_{\text {fast }}(Q)$ of the fast process. Bulk $(\bigcirc, \boldsymbol{\odot}), 1000 \AA(\square, \boldsymbol{\square})$, $400 \AA(\boldsymbol{\square}, \boldsymbol{\nabla})$. Open and closed symbols correspond to $\Gamma$ and $A_{\text {fast }}(Q)$, respectively.

$A_{\text {fast }}(Q)$ are plotted against the inverse of $T$ in Fig. 8 for the bulk and the 1000 and $400 \AA$ films. The relaxation rate $\Gamma$ is almost independent of temperature for the bulk and the 1000 and $400 \AA$ films, showing that the activation energy of the fast process is almost zero or very small. This result implies that the fast process is a localized motion within a potential well (cage motion) [35], which is also supported by the fact that the relaxation rate $\Gamma$ is independent of $Q$ (Ref. [52]) for all the samples as shown in Fig. 9. On the other hand, the fraction $A_{\text {fast }}(Q)$ of the fast process increases with temperature, which must correspond to the increase in the amplitude of the motion because the fast process is a localized motion in a potential well. What we have to emphasize here is that the fraction $A_{\text {fast }}(Q)$ decreases with the film thickness. The film thickness dependence of $A_{\text {fast }}(Q)$ is shown in Fig. 10. As discussed above, the boson peak intensity $G_{\mathrm{B}}(\omega)$ also decreases with the film thickness, which is indicated in Fig. 10. The film thickness dependence is very similar between $A_{\text {fast }}(Q)$ and $G_{\mathrm{B}}(\omega)$, suggesting that the two modes are caused by the same origin. In addition, both of the relaxation rate $\Gamma$ of the fast proces $(\sim 1 \mathrm{meV})$ and the characteristic energy of the boson peak (the peak energy $\sim 1.5 \mathrm{meV}$ ) are close to each other and are independent of the film thickness. This also suggests that the origin of the two modes are the same.

In the discussion on the boson peak, we assumed that the decrease in the density of phonon states $G_{\mathrm{B}}(\omega)$ was caused

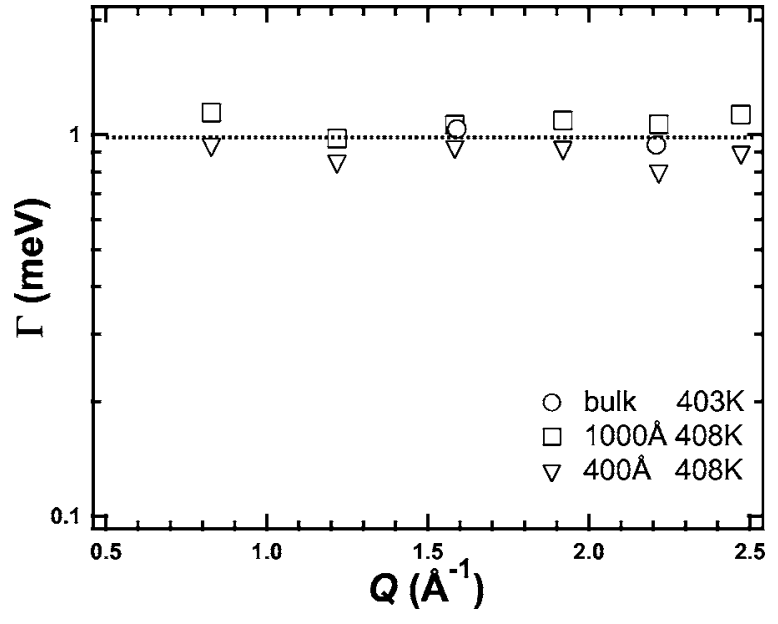

FIG. 9. $Q$ dependence of relaxation rate $\Gamma$ of the fast process. Bulk (○), $1000 \AA(\square)$, and $400 \AA(\nabla)$.

by the decrease of defects or voids in the hard layer at the interface. This picture would explain the decrease of the fraction of the fast process $A_{\text {fast }}(Q)$ as follows. Assuming that the fast process is originated from the same defects or voids as the boson peak, the decrease of them also induces the decrease in the fraction of the fast process. The fact that the the boson peak energy and the relaxation rate $\Gamma$ of the fast process are independent of the film thickness would predict that the defects or voids in the hard layer decreases in the number but not so different from the bulk in nature, such as the distributions of size and shape. It is, therefore, considered at the moment that the hard layer or the ordered layer is one of the most plausible causes for the decrease of a boson peak mode as well as the fast process.

\section{CONCLUSION}

In this paper, we have studied the low-energy excitations and the picosecond fast process in polystyrene thin supported films using inelastic and quasielastic neutron scattering in a temperature range from 11 to $430 \mathrm{~K}$, covering the bulk glass transition temperature $T_{\mathrm{g}}$. We found in the analysis of elastic

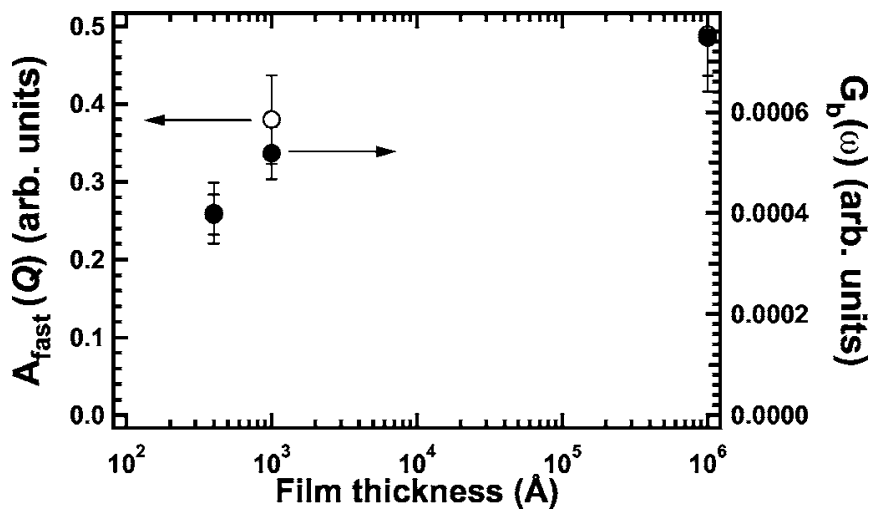

FIG. 10. Thickness dependence of a fraction of the fast process $A_{\text {fast }}(Q)(\bigcirc)$ and density of phonon states of the boson peak mode $G_{\mathrm{B}}(\omega)(\bullet)$. 
scattering that the mean square displacement $\left\langle u^{2}\right\rangle$ decreased with the film thickness in the whole temperature range, showing the hardening in the thinner films. In the inelastic scattering, we also found that the density of phonon states $G(\omega)$ decreased with the film thickness in both the Debye mode and the boson peak mode although the boson peak energy was unaltered. Furthermore, in the quasielastic scattering, the fraction of the picosecond fast process $A_{\text {fast }}(Q)$ decreased with the film thickness without changing the relaxation rate $\Gamma$. The thickness dependence of $A_{\text {fast }}(Q)$ is very similar to that of the density of phonon states of the boson peak $G_{\mathrm{b}}(\omega)$, suggesting that the boson peak and the fast process are originated from the same molecular origin. We have examined two possibilities for the hardening: chain confinement effects in a thin film and the hard layer at the interface. Assuming that the defects or voids are the molecular origin for the both modes, the decrease in the number of defects or voids in the hard layer without changing the nature could explain all the observations in the experiments. It is, therefore, concluded at the moment that the hard layer at the interface is one of the most plausible reasons for the hardening with decreasing the film thickness.
[1] Polymer Surface, Interfaces and Thin Films, edited by A. Karim and S. Kumar (World Scientific, Singapore, 2000).

[2] Polymer Surfaces, From Physics to Technology, edited by F. Garbassi, M. Morra, and E. Occhiello (Wiley, Chichester, 1998).

[3] J. L. Keddie, R. A. L. Jones, and R. A. Cory, Europhys. Lett. 27, 59 (1994).

[4] J. L. Keddie, R. A. L. Jones, and R. A. Cory, Faraday Discuss. 98, 219 (1994).

[5] S. Kawana and R. A. L. Jones, Phys. Rev. E 63, 021501 (2001).

[6] J. A. Forrest, K. Dalnoki-Veress, J. R. Stevens, and J. R. Dutcher, Phys. Rev. Lett. 77, 2002 (1996).

[7] K. Dalnoki-Veress, J. A. Forrest, C. Murray, C. Gigault, and J. R. Dutcher, Phys. Rev. E 63, 031801 (2001).

[8] J. Mattsson, J. A. Forrest, and L. Borjsson, Phys. Rev. E 62, 5187 (2000).

[9] G. Beaucage, R. Composto, and R. S. Stein, J. Polym. Sci., Part B: Polym. Phys. 31, 319 (1993).

[10] T. Miyazaki, K. Nishida, and T. Kanaya, Phys. Rev. E 69, 022801 (2004).

[11] T. Miyazaki, K. Nishida, and T. Kanaya, Phys. Rev. E 69, 061803 (2004).

[12] W. E. Wallace, N. C. Beck Tan, and W. L. Wu, J. Chem. Phys. 108, 3798 (1998).

[13] G. B. DeMaggio, W. E. Frieze, D. W. Gidley, M. Zhu, H. A. Hristov, and A. F. Yee, Phys. Rev. Lett. 78, 1524 (1997).

[14] K. Fukao and Y. Miyamoto, Phys. Rev. E 61, 1743 (2001).

[15] K. Fukao and Y. Miyamoto, Phys. Rev. E 64, 011803 (2001).

[16] T. Kajiyama, K. Tanaka, and A. Takahara, Polymer 39, 4665 (1998).

[17] T. Kajiyama, K. Tanaka, N. Satomi, and A. Takahara, Macromolecules 31, 5150 (1999).

[18] N. Satomi, A. Takahara, and Kajiyama, Macromolecules 32, 4474 (1999).

[19] M. Yu. Efremov, E. A. Olson, M. Zhang, Z. Zhang, and L. H. Allen, Macromolecules 37, 4607 (2004).

[20] M. Yu. Efremov, E. A. Olson, M. Zhang, Z. Zhang, and L. H. Allen, Phys. Rev. Lett. 91, 085703 (2003).

[21] F. L. Pratt, T. Lancaster, M. L. Brooks, S. J. Blundell, T. Prokscha, E. Morenzoni, A. Suter, H. Luetkens, R. Khasanov, R. Scheuermann, U. Zimmermann, K. Shinotsuka, and H. E. Assender, Phys. Rev. B 72, 121401(R) (2005).

[22] W. J. Orts, J. H. vanZanten, W. L. Wu, and S. K. Satija, Phys.
Rev. Lett. 71, 867 (1993)

[23] T. Kanaya, T. Miyazaki, R. Inoue, and K. Nishida, Phys. Status Solidi B 242, 595 (2005).

[24] J. A. Forrest, C. Svanberg, K. Révész, M. Rodahl, L. M. Torell, and B. Kasemo, Phys. Rev. E 58, R1226 (1998).

[25] H. Kim, A. Rühm, L. B. Lurio, J. K. Basu, J. Lal, D. Lumma, S. G. J. Mochrie, and S. K. Sinha, Phys. Rev. Lett. 90, 068302 (2003).

[26] C. L. Soles, J. F. Douglas, W. L. Wu, and R. M. Dimeo, Phys. Rev. Lett. 88, 037401 (2002).

[27] C. L. Soles, J. F. Douglas, W. Wu, H. Peng, and D. W. Gidley, Macromolecules 37, 2890 (2004).

[28] B. Frick, K. Dalnoki-Veress, J. A. Forrest, J. Dutcher, C. Murray, and A. Higgins, Eur. Phys. J. D 12, 93 (2003).

[29] R. Inoue, T. Kanaya, K. Nishida, I. Tsukushi, and K. Shibata, Phys. Rev. Lett. 95, 056102 (2005).

[30] K. Inoue, Y. Ishikawa, N. Watanabe, K. Kaji, Y. Kiyanagi, H. Iwasa, and M. Kohgi, Nucl. Instrum. Methods Phys. Res. A 238, 401 (1984).

[31] K. Inoue, T. Kanaya, S. Ikeda, K. Kaji, K. Shibata, M. Misawa, and Y. Kiyanagi, J. Chem. Phys. 95, 5333 (1991).

[32] U. Buchenau, M. Prager, N. Nücker, A. J. Dianoux, N. Ahmad, and W. A. Phillips, Phys. Rev. B 34, 5665 (1986).

[33] B. Frick and D. Richter, Phys. Rev. B 47, 14795 (1993).

[34] B. Frick, U. Buchenau, and D. Richter, Colloid Polym. Sci. 274, 413 (1995).

[35] T. Kanaya, T. Kawaguchi, and K. Kaji, J. Chem. Phys. 104, 3841 (1996).

[36] U. Buchenau, C. Schönfeld, D. Richter, T. Kanaya, K. Kaji, and R. Wehrmann, Phys. Rev. Lett. 73, 2344 (1994).

[37] R. Zorn, A. Arbe, J. Colmenero, B. Frick, D. Richter, and U. Buchenau, Phys. Rev. E 52, 781 (1995).

[38] W. Marshall and S. W. Lovesey, Theory of Thermal Neutron Scattering (Clarendon, Oxford, 1971).

[39] C. L. Choy, R. G. Hunt, and G. L. Salinger, J. Chem. Phys. 52, 3629 (1970).

[40] J. Brandrup, E. H. Immergut, and E. A. Grulke, Polymer Handbook (John Wiley Sons, New York, 1998).

[41] R. Zorn, L. Hartmann, B. Frick, D. Richter, and F. Kremer, J. Non-Cryst. Solids 307, 547 (2002).

[42] R. Zorn, B. Frick, L. Hartmann, D. Richter, F. Kremer, A. Schönhals, and D. Richter, Physica D 350, e1115 (2004).

[43] J. Schuyer, J. Polym. Sci. 36, 475 (1959).

[44] M. K. Sanyal, J. K. Nasu, A. Datta, and S. Banerjee, Europhys. 
INOUE et al.

PHYSICAL REVIEW E 74, 021801 (2006)

Lett. 36, 265 (1996).

[45] J. Kraus, P. Mueller-Buschbaum, T. Kuhlmann, D. W. Schubert, and M. Stamm, Europhys. Lett. 49, 210 (2000).

[46] M. Mukherjee, M. Bhattacharya, M. K. Sanyal, T. Geue, J. Grenzer, and U. Pietsch, Phys. Rev. E 66, 061801 (2002).

[47] T. Nakayama, Phys. Rev. Lett. 80, 1244 (1998).

[48] Y. Inamura, M. Arai, M. Nakamura, T. Otomo, N. Kitamura, S. M. Bennington, A. C. Hannon, and U. Buchenau, J. Non-
Cryst. Solids 293, 389 (2001).

[49] S. P. Das, Phys. Rev. E 59, 3870 (1999).

[50] A. V. Granato, Physica B 220, 270 (1996).

[51] T. Kanaya, T. Kawaguchi, and K. Kaji, J. Chem. Phys. 105, 4342 (1996).

[52] T. Kanaya, T. Kawaguchi, and K. Kaji, J. Chem. Phys. 98, 8262 (1993). 